\title{
Squamous Cell Transformation of Primary Lung Adenocarcinoma in a Patient With EML4-ALK Fusion Variant 5 Refractory to ALK Inhibitors
}

Jay Gong, MS a,b; Jeffrey P. Gregg, MDc; Weijie Ma, MDa,b; Ken Yoneda, MDd; Elizabeth H. Moore, MDe; Megan E. Daly, MDf; Yanhong Zhang, MD, MSs; Melissa J. Williams, MD'; and Tianhong Li, MD, PhDa,b

\begin{abstract}
Histologic transformation from adenocarcinoma to squamous cell carcinoma in lung cancer has not been reported as a mechanism of resistance to ALK inhibition. This report describes the clinical course of a female former light smoker with metastatic lung adenocarcinoma whose tumor underwent histologic transformation from a welldifferentiated lung adenocarcinoma to a well-differentiated lung squamous cell carcinoma in the same location at the left mainstem bronchus while maintaining the ALK fusion oncogene without any resistance mutations. After experiencing disease progression while on crizotinib, the patient participated in clinical trials that provided early access to the novel ALK inhibitors ceritinib and alectinib before they were commercially available. Tumor recurrence occurred at the primary and metastatic central nervous system sites (ie, brain and spine). At tumor progression, liquid biopsy and tumor genomic profiling of plasma cell-free DNA next-generation sequencing (NGS) provided an accurate diagnosis with a short turnaround time compared with the tissue-based targeted capture NGS. The patient received several courses of radiation primarily to the brain and spine during her disease course. Her disease did not respond to the immune checkpoint inhibitor nivolumab, and she died on home hospice approximately 4 years after diagnosis. This case supports the importance of both histopathologic assessment and comprehensive genomic profiling in selecting appropriate treatment for patients with refractory, metastatic, ALK oncogene-driven nonsmall cell lung cancer. Use of symptom-directed radiation in tandem with ALK inhibitors contributed to the disease and symptomatic control and prolonged survival in this patient.
\end{abstract}

J Natl Compr Canc Netw 2019;17(4):297-301 doi: 10.6004/jnccn.2019.7291

\footnotetext{
aDivision of Hematology/Oncology, Department of Internal Medicine, University of California Davis School of Medicine, Sacramento; ${ }^{b}$ University of California Davis Comprehensive Cancer Center, Sacramento; 'Department of Pathology and Laboratory Medicine and Genomic Shared Resource, ${ }^{\mathrm{d}}$ Division of Pulmonary, Critical Care, and Sleep Medicine, Department of Internal Medicine, and 'Department of Radiology, University of California Davis School of Medicine, Sacramento; 'Department of Radiation Oncology, University of California Davis Comprehensive Cancer Center, Sacramento; ${ }^{9}$ Department of Pathology, Kaiser Permanente Vallejo Medical Center, Vallejo; and ${ }^{\text {h}}$ Sutter Davis Medical Group, Davis, California.
}

\section{Background}

Originally discovered in lymphomas, ${ }^{1}$ the $A L K$ fusion oncogene with gain-of-function cytoplasmic tyrosine kinase activity has since been identified as an oncogenic driver in $3 \%$ to $10 \%$ of patients with non-small cell lung cancer (NSCLC). ${ }^{2,3}$ FDA approval of crizotinib, a small molecule ALK tyrosine kinase inhibitor (TKI), only 3 years after the discovery of the $A L K$ fusion oncogene represents one of the most rapid bench-tobedside translational advances in the history of targeted cancer therapy. ${ }^{4}$ Among the many fusion partners that have been reported, EML4 is the most common gene partner of $A L K$ through a paracentric inversion of chromosome $2 \operatorname{inv}(2)$ (p21;p23). We previously reported that in a cohort of 200 NSCLC specimens, the EML4$A L K$-positive transcripts included 109 variant 1 (V1; $54.5 \%), 20 \mathrm{~V} 2$ (10.0\%), $68 \mathrm{~V} 3$ (34.0\%), and 3 V5a (1.5\%) variants. ${ }^{5}$ Most $(\mathrm{n}=188 ; 94.0 \%) E M L 4-A L K$-positive NSCLC tumors had adenocarcinoma histology. ALK expression level varied significantly among different $E M L 4-A L K$ variants and individual tumors, with V3 having the lowest $A L K$ expression level by reverse transcription polymerase chain reaction (RT-PCR) assay. ${ }^{5,6}$ Although $E M L 4-A L K \mathrm{~V} 1$ has been shown to be most common in Caucasians, V3 has been shown to be most common in the Chinese population. ${ }^{7}$ Recent studies have shown that EML4-ALKV1 and V3 may have different sensitivity to ALK inhibitors and different resistant mutations. ${ }^{8,9}$ Little is known regarding whether rare variants other than $E M L 4-A L K \mathrm{~V} 1, \mathrm{~V} 3$, or different fusion partners affect the clinical benefit of ALK inhibitors.

Histologic transformation from lung adenocarcinoma (LUAD) to small cell lung carcinoma (SCLC) or large cell neuroendocrine tumor has been associated with resistance to EGFR ${ }^{10-12}$ and ALK TKIs. ${ }^{13}$ Although histologic transformation from LUAD to lung squamous

See JNCCN.org for supplemental online content. 
cell carcinoma (LUSC) has been reported as a mechanism of resistance to EGFR inhibition, ${ }^{14,15}$ it has not been reported for ALK inhibition. This report describes the clinical course of a female former light smoker with metastatic LUAD whose tumor underwent histologic transformation from a well-differentiated LUAD to a well-differentiated LUSC in the same location in the left mainstem bronchus while maintaining the $A L K$ fusion as the driver oncogene without any known resistance mutation detected by clinical next-generation sequencing (NGS) assays.

\section{Materials and Methods}

The patient provided consent to participate in an Institutional Review Board-approved study (University of California, Davis, \#226210), as well as consent for publication. The clinical history, radiographic images, and histologic and immunohistochemistry stains were performed as standard of care clinical tests at our institution. Fluorescence in situ hybridization (FISH) assay was performed using the Vysis ALK Break Apart FISH Probe Kit (Abbott Laboratories) at a commercial CLIA-certified laboratory. A board-certified radiologist (E.H.M) and pathologists (J.P.G., Y.Z.) selected the images. Plasma cell-free DNA (cfDNA) and tumor specimens were subjected to the hybridization capture-based NGS (FoundationACT for blood and FoundationOne for tissue) assays performed at a CLIA-certified, College of American Pathologists (CAP)-accredited, New York Stateapproved laboratory (Foundation Medicine, Inc). ${ }^{16,17}$

\section{Case Presentation}

In January 2013, a 60-year-old Hispanic female former light smoker (4 pack-year history who quit 33 years prior to diagnosis) was diagnosed with LUAD with a lingular primary, at least 9 subcentimeter metastases to the brain, and with additional lesions in the liver, spleen, and bones. The patient underwent bronchoscopy, which revealed diffuse submucosal hemorrhage in the left mainstem bronchus and a tumor obstructing approximately $30 \%$ to $50 \%$ of the left upper lobe which corresponded to the chest CT findings (Figure 1A). The diagnostic tumor specimen tested negative for EGFR L858R, exon 19 deletion, and T790M mutations by RTPCR, but positive for $A L K$ gene rearrangement by FISH, without any information on the fusion partner. Figure 2 and supplemental eTable 1 (available with this article at JNCCN.org) summarize the major diagnostic and treatment course of this patient over almost 4 years (in order): $30 \mathrm{~Gy}$ of palliative whole-brain radiation therapy (WBRT) in 10 daily fractions; crizotinib for 13 months; 30 Gy of palliative external-beam RT (EBRT) to T11-L1 in 10 daily fractions; ceritinib for 10 months; alectinib for 7 months; 30 Gy of palliative EBRT to T4-T9 in 10 fractions. The patient also received zoledronic acid first and then denosumab for approximately 2 years to prevent fracture, spinal cord compression, or the need for RT.

In July 2016, the patient presented with chest and back pain and dyspnea. Chest CT showed disease progression with confluent soft tissue attenuation and adenopathy within the subcarinal space that invaded and occluded the left mainstem bronchus and extended into the left upper and lower lobes with postobstructive consolidation (Figure 1B), small left pleural effusion, right-sided mediastinal adenopathy, and new pleural nodularity along the medial aspect of the right hemithorax. A bronchoscopy on August 2, 2016, revealed that the left mainstem bronchus was completely obliterated by friable tissue. Cryoablation was performed with restoration of approximately $50 \%$ patency. Pathology showed invasive well-differentiated LUSC (Figure 1D) that was TTF-1-negative (Figure 1F) and P40-positive (data not shown) compared with the original LUAD (Figure 1C) that was TTF-1-positive (Figure 1E). Tumor genomic profiling assay of this tumor and plasma cfDNA revealed a rare driver oncogene, EML4-ALK V5 (supplemental eTable 2). Other notable genomic alterations in the tumor specimen included PTEN loss of exon 1, CDKN2A and CDKN2B loss, and FAS loss. The tumor had high tumor mutational burden (TMB) of 15.17 mutations per megabase (Muts/Mb), and positive PD-Ll expression with a tumor proportion score of $3 \%$ by PD-L1 IHC 22C3 pharmDx assay (Agilent).

Due to her poor performance status, the patient was deemed not a candidate for chemotherapy. Instead, she received 30 Gy of EBRT to the left hilum in 10 daily fractions, and showed symptomatic improvement. Shortly thereafter, brain MRI revealed diffuse progression of intracranial metastases. After extensive discussion, an additional course of WBRT to $25 \mathrm{~Gy}$ in 10 fractions was performed. She received pembrolizumab for 2 cycles without any significant clinical improvement, and died after enrolling in home hospice care several weeks later.

\section{Discussion}

NGS for Detecting Rare Variants of EML4-ALK Fusion Oncogene in Tissue and Blood

Histology assessment of lung cancer at diagnosis is important for selecting chemotherapy and molecularly targeted therapy. ${ }^{18}$ Currently, testing for $A L K$ rearrangements is recommended for patients diagnosed with adenocarcinoma and mixed lung cancers with an adenocarcinoma component, regardless of clinical characteristics. ${ }^{19}$ At initial diagnosis in early 2013, our patient was found to have $A L K$-rearranged LUAD by the ALK break-apart FISH assay. However, FISH is less sensitive or unable to detect the fusion variants and partners compared with NGS. ${ }^{20}$

Despite exceptional tumor response rates and improved survivals, almost all patients with $A L K$-rearranged NSCLC eventually develop acquired resistance to the 

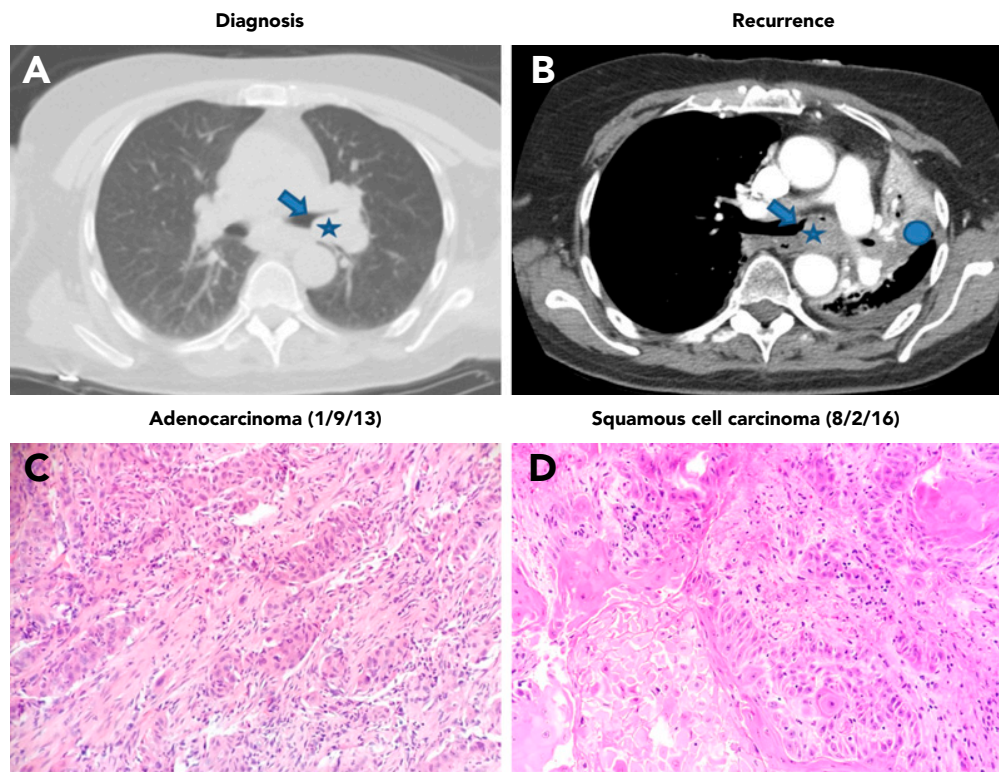

Squamous cell carcinoma $(8 / 2 / 16)$
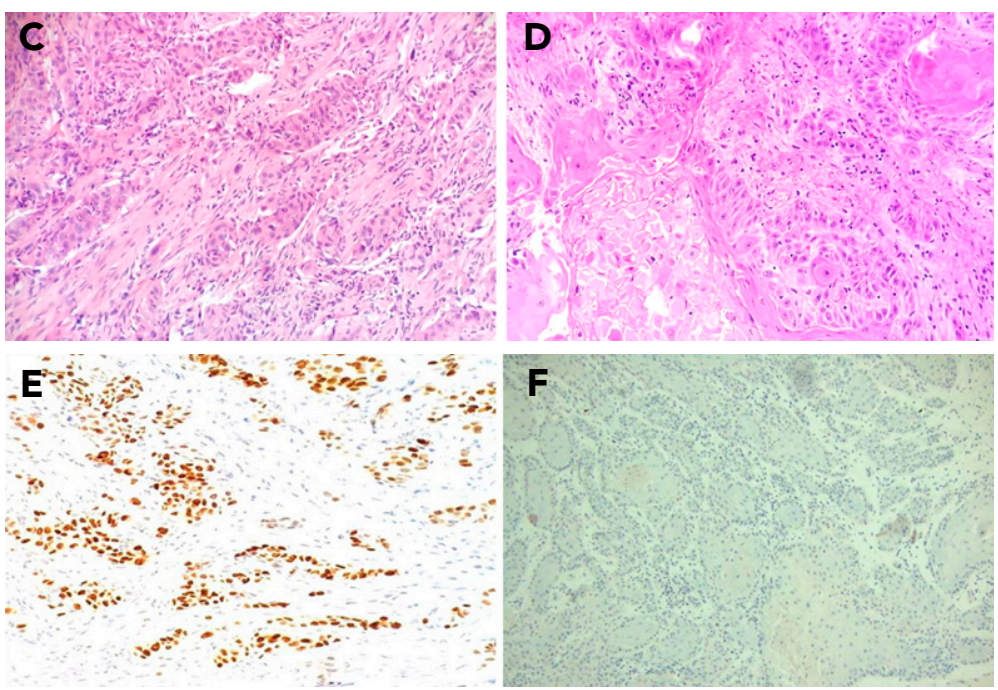

Figure 1. Radiographic imaging, histomorphology, and immunohistochemical staining of the initial lung adenocarcinoma (LUAD) and lung squamous cell transformation at the same tumor site. (A) Chest CT showed the tumor at the left upper mainstem (star) obstructing the left mainstem bronchus (arrow), causing (B) left upper lobe collapse (solid circle). (C-F) Histologic assessment of different lung pathologies. (C) Hematoxylin-eosin staining revealed LUAD at diagnosis and (D) invasive, well-differentiated, squamous cell carcinoma approximately 3.5 years later at the same location. (E) TTF-1 immunohistochemical staining was positive in LUAD and (E) negative in lung squamous cell carcinoma. The adenocarcinoma also stained positive for CK7 and negative for CK20 and synaptophysin (data not shown). The squamous cell cancer stains were positive for $\mathrm{P} 40$ (data not shown) (original magnification, $\times 20$ for all).

ALK TKIs. ${ }^{21}$ Both ALK-dependent and -independent mechanisms can contribute to the development of resistance mechanisms to ALK inhibitors, which include the presence of a second-site mutation in the tyrosine kinase domain of $A L K$ gene in $20 \%$ to $30 \%$ of cases, $A L K$ amplification, $M E T$ proto-oncogene amplification, activation of bypass pathways, and, rarely, histologic transformation..$^{22}$ In the present case, we found histologic transformation from well-differentiated LUAD to well-differentiated keratinizing LUSC in the same location of the left main bronchus site in August 2016. We could not determine the $A L K$ fusion partner and variant of the original tumor, because there was insufficient tumor specimen remaining from the initial diagnosis. Detection of the oncogenic EML4-ALKV5 without any known molecular resistance mutations in both the tumor and blood after disease had progressed on 3 ALK inhibitors suggests histologic transformation might be a resistance mechanism to the ALK inhibitors.
Potential Mechanisms and Clinical Implication of Histologic Transformation

Histologic transformation from LUAD to SCLC has been reported in EGFR-mutant and $A L K$-rearranged LUAD. ${ }^{23-25}$ Recent reports suggest the transformation occurs from early clonal evolution and coexpression of molecular markers (such as loss of RB and P53) found in the transformed histology (ie, SCLC). ${ }^{26,27}$ These transformed tumors behave biologically like SCLC and have a treatment response and survival comparable to those of typical SCLC. ${ }^{28}$

We do not know the timing and the mechanism controlling the histologic transformation at the same tumor site after ALK inhibitors and RT. Similar to our reported results, FoundationOne identified several genomic alterations, including PTEN loss, CDKN2A and CDKN2B loss, FAS loss, and FBXW7 E113D - subclonal (supplemental eTable 2), that are frequently reported in LUSC but not LUAD. In addition, the tumor had a high TMB of 15.17 Muts/Mb. LUSC shows a higher TMB 


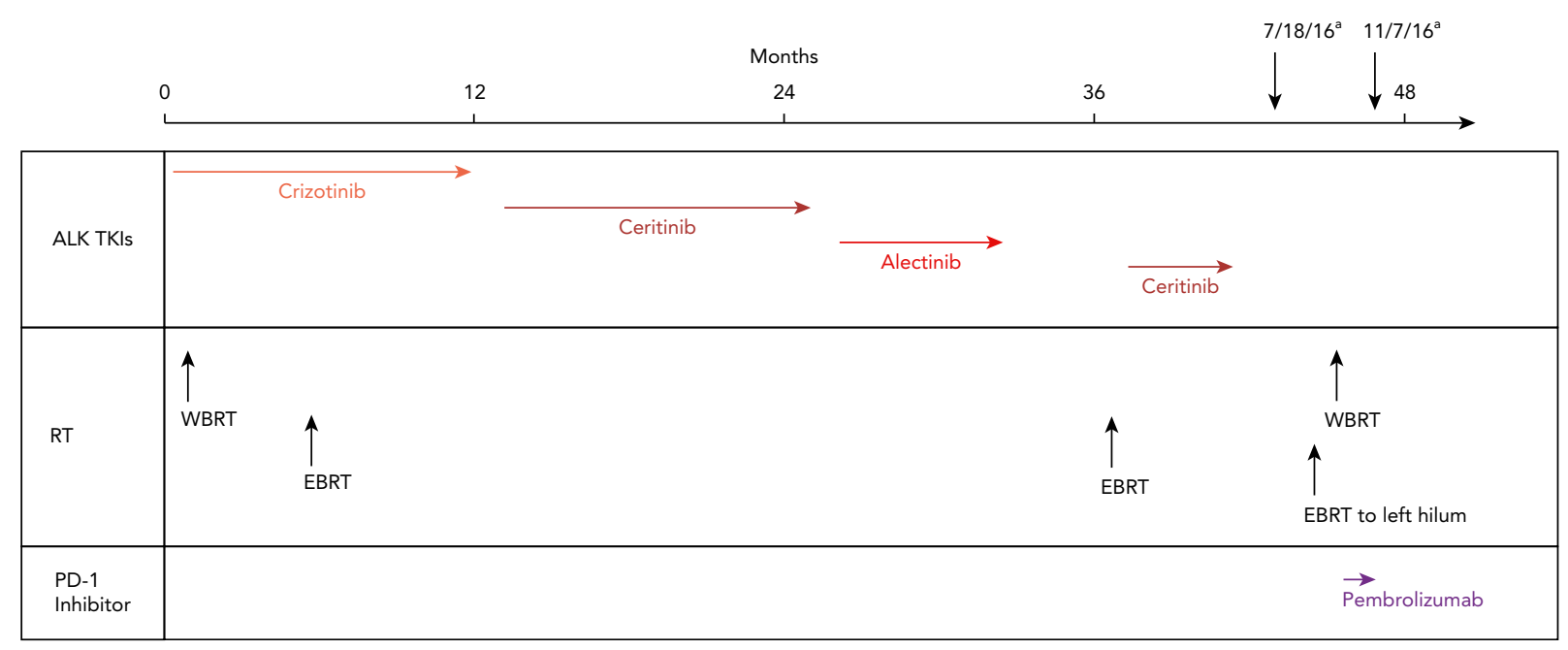

Figure 2. Summary of the patient's clinical course for both extracranial and central nervous system tumors. The patient was diagnosed in January 2013 and passed away in December 2016. Arrowheads indicate time points for each intervention.

Abbreviations: EBRT, external-beam radiation therapy; TKI, tyrosine kinase inhibitor; WBRT, whole-brain radiation therapy.

aBlood draws were performed, and EML4-ALK variant 5 was the only genomic alteration detected in the plasma cell-free DNA by FACT (supplemental eTable 2).

than LUAD (9.6 vs 6.3 Muts/Mb, respectively) in a large human genomic database. ${ }^{29}$ Unfortunately, our patient was paralyzed from spine metastasis and had significant physical deconditioning, rendering her unsuitable for chemotherapy. Although no adenocarcinoma or small cell component was identified in the resistant tumor with squamous cell transition, intertumor heterogeneity or sampling artifacts cannot be completely ruled out. Tumor genomic profiling identified the rare $E M L 4-A L K \mathrm{~V} 5$ as the only genomic alteration in plasma cfDNA at the time of tumor sampling of the squamous cell transition and 4 months later, and did not reveal any other resistant mutations to ALK inhibitors or other alterations (supplemental eTable 2), suggesting that the $A L K$-rearranged gene was the dominant oncogenic driver. In support of this, we recently observed that mice bearing xenografts established from the tumor biopsy performed at the time of the squamous cell transformation have significant tumor shrinkage in response to a newer ALK inhibitor brigatinib (data not shown).

\section{Multidisciplinary Management for Patients With NSCLC and ALK Fusion Oncogenes}

Patients with $A L K$-rearranged NSCLC have been found to have a higher incidence of brain metastasis either at initial diagnosis or after control of systemic disease with crizotinib. $^{21,30}$ In approximately half of the patients treated with crizotinib, brain metastases develop or progress. Our patient was diagnosed with LUAD with a lingular primary with high TMB in both central nervous system and extracranial sites. After experiencing disease progression on crizotinib, our patient was enrolled in clinical trials which enabled her to receive the secondgeneration ALK inhibitors ceritinib and alectinib before FDA approval. RT has been increasingly integrated into the clinical management of patients with metastatic NSCLC, both for symptom relief and for local disease control of oligometastatic progression while on otherwise successful systemic therapy, particularly in patients with oncogene-driven NSCLC on molecularly targeted therapy. ${ }^{31,32}$ Our patient received 5 courses of palliative RT during her disease course. Use of symptom-directed RT in this patient in tandem with ALK inhibitors offered disease and symptom control that may have improved her survival. Although ICIs have revolutionized the treatment of patients with advanced NSCLC, and consistent with previous reports, ${ }^{33,34}$ we did not observe any clinical response to pembrolizumab despite squamous cell transformation, positive PD-L1 immunohistochemical expression, and high TMB. Further study is needed to define the role of ICIs in $A L K$-rearranged NSCLC.

\section{Conclusions}

To our knowledge, this is the first report of histologic transformation from LUAD to LUSC at the same tumor location while maintaining the $A L K$-rearranged driver oncogene. Tumor genomic profiling using NGS can identify the rare EML4-ALKV5 fusion gene. Liquid biopsy and plasma cfDNA offer a quick and reliable alternative to invasive tissue biopsy to detect the rare driver oncogene and explore the resistance mechanisms. There are unmet needs to delineate the molecular mechanisms underlying historical transformation from LUAD to LUSC, to develop novel strategies to overcome the resistance to 
ALK TKIs with activity for both extracranial and intracranial metastases, and to develop effective cancer immunotherapy strategies for $A L K$-rearranged lung cancer.

Submitted September 29, 2018; accepted for publication February 26, 2019.

Disclosures: Dr. Gregg has disclosed that he is a consultant for Foundation Medicine; receives honoraria from and serves on a scientific advisory board for AstraZeneca; and is a consultant for Bristol-Myers Squibb. Dr. Yoneda has disclosed that he is a scientific advisor for AstraZeneca and Guardant Health Dr. Daly has disclosed that she receives research funding from EMD Serono. Dr. Li has disclosed that she is a scientific advisor for Foundation Medicine, Takeda, and PUMA, and receives grant/research support from Foundation Medicine, Pfizer, AstraZeneca, Hengrui, and Eureka. The remaining authors have disclosed that they have not received any financial consideration from any person or organization to support the preparation, analysis, results, or discussion of this article.

Correspondence: Tianhong Li, MD, PhD, University of California Davis Comprehensive Cancer Center, 4501 X Street, Suite 3016, Sacramento, CA 95817. Email: thli@ucdavis.edu

\section{References}

1. Morris SW, Kirstein MN, Valentine MB, et al. Fusion of a kinase gene, ALK, to a nucleolar protein gene, NPM, in non-Hodgkin's lymphoma. Science 1994;263:1281-1284.

2. Soda $\mathrm{M}$, Choi $\mathrm{YL}$, Enomoto $\mathrm{M}$, et al. Identification of the transforming EML4-ALK fusion gene in non-small-cell lung cancer. Nature 2007;448: 561-566.

3. Lin E, Li L, Guan Y, et al. Exon array profiling detects EML4-ALK fusion in breast, colorectal, and non-small cell lung cancers. Mol Cancer Res 2009; 7:1466-1476.

4. Malik SM, Maher VE, Bijwaard KE, et al. U.S. Food and Drug Administration approval: crizotinib for treatment of advanced or metastatic nonsmall cell lung cancer that is anaplastic lymphoma kinase positive. Clin Cancer Res 2014;20:2029-2034.

5. Li T, Maus MK, Desai SJ, et al. Large-scale screening and molecular characterization of EML4-ALK fusion variants in archival non-small-cell lung cancer tumor specimens using quantitative reverse transcription polymerase chain reaction assays. J Thorac Oncol 2014;9:18-25.

6. Woo CG, Seo S, Kim SW, et al. Differential protein stability and clinical responses of EML4-ALK fusion variants to various ALK inhibitors in advanced ALK-rearranged non-small cell lung cancer. Ann Oncol 2017;28:791-797.

7. Tian $\mathrm{HX}$, Zhang XC, Yang JJ, et al. Clinical characteristics and sequence complexity of anaplastic lymphoma kinase gene fusions in Chinese lung cancer patients. Lung Cancer 2017;114:90-95.

8. Lin JJ, Zhu VW, Yoda S, et al. Impact of EML4-ALK variant on resistance mechanisms and clinical outcomes in ALK-positive lung cancer. J Clin Oncol 2018;36:1199-1206.

9. Ou SH, Schrock AB, Gowen K, et al. Association of ALK resistance mutations by EML4-ALKvariant (v3 vs. non-v3) in ALK+ non-small cell lung cancer (NSCLC) [abstract]. J Clin Oncol 2017;35(Suppl):Abstract 9010.

10. Kim WJ, Kim S, Choi H, et al. Histological transformation from non-small cell to small cell lung carcinoma after treatment with epidermal growth factor receptor-tyrosine kinase inhibitor. Thorac Cancer 2015;6:800-804.

11. Moriya R, Hokari S, Shibata $S$, et al. Histological transformation to large cell neuroendocrine carcinoma from lung adenocarcinoma harboring an EGFR mutation: an autopsy case report. Intern Med 2017;56:2013-2017.

12. Hui M, Uppin SG, Stalin BJ, et al. Histological transformation of adenocarcinoma to small cell carcinoma lung as a rare mechanism of resistance to epidermal growth factor receptor-tyrosine kinase inhibitors: report of a case with review of literature. Lung India 2018;35:160-163.

13. Levacq D, D'Haene N, de Wind R, et al. Histological transformation of ALK rearranged adenocarcinoma into small cell lung cancer: a new mechanism of resistance to ALK inhibitors. Lung Cancer 2016;102:38-41.

14. Levin PA, Mayer M, Hoskin S, et al. Histologic transformation from adenocarcinoma to squamous cell carcinoma as a mechanism of resistance to EGFR inhibition. J Thorac Oncol 2015;10:e86-88.

15. Park HK, Seo Y, Choi YL, et al. Metastatic squamous cell carcinoma from lung adenocarcinoma after epidermal growth factor receptor tyrosine kinase inhibitor therapy. J Pathol Transl Med 2017;51:441-443.

16. Frampton GM, Fichtenholtz A, Otto GA, et al. Development and validation of a clinical cancer genomic profiling test based on massively parallel DNA sequencing. Nat Biotechnol 2013;31:1023-1031.

17. Clark TA, Chung JH, Kennedy M, et al. Analytical validation of a hybrid capture-based next-generation sequencing clinical assay for genomic profiling of cell-free circulating tumor DNA. J Mol Diagn 2018;20:686-702.
18. Li T, Kung HJ, Mack PC, et al. Genotyping and genomic profiling of nonsmall-cell lung cancer: implications for current and future therapies. J Clin Oncol 2013;31:1039-1049.

19. Lindeman NI, Cagle PT, Aisner DL, et al. Updated molecular testing guideline for the selection of lung cancer patients for treatment with targeted tyrosine kinase inhibitors: guideline from the College of American Pathologists, the International Association for the Study of Lung Cancer, and the Association for Molecular Pathology. J Thorac Oncol 2018;13:323-358.

20. Ali SM, Hensing $T$, Schrock AB, et al. Comprehensive genomic profiling identifies a subset of crizotinib-responsive ALK-rearranged non-small cell lung cancer not detected by fluorescence in situ hybridization. Oncologist 2016;21:762-770

21. Shaw AT, Yeap BY, Mino-Kenudson M, et al. Clinical features and outcome of patients with non-small-cell lung cancer who harbor EML4-ALK. J Clin Oncol 2009;27:4247-4253.

22. Katayama R, Shaw AT, Khan TM, et al. Mechanisms of acquired crizotinib resistance in ALK-rearranged lung cancers. Sci Transl Med 2012;4:120ra17.

23. Sequist LV, Waltman BA, Dias-Santagata D, et al. Genotypic and histological evolution of lung cancers acquiring resistance to EGFR inhibitors. Sci Transl Med 2011;3:75ra26.

24. Norkowski E, Ghigna MR, Lacroix L, et al. Small-cell carcinoma in the setting of pulmonary adenocarcinoma: new insights in the era of molecular pathology. J Thorac Oncol 2013;8:1265-1271.

25. Fujita S, Masago K, Katakami N, et al. Transformation to SCLC after treatment with the ALK inhibitor alectinib. J Thorac Oncol 2016;11:e67-72.

26. Niederst MJ, Sequist LV, Poirier JT, et al. RB loss in resistant EGFR mutant lung adenocarcinomas that transform to small-cell lung cancer. Nat Commun 2015;6:6377.

27. Lee JK, Lee J, Kim S, et al. Clonal history and genetic predictors of transformation into small-cell carcinomas from lung adenocarcinomas. J Clin Oncol 2017;35:3065-3074.

28. Ferrer L, Giaj Levra M, Brevet M, et al. A brief report of transformation from NSCLC to SCLC: molecular and therapeutic characteristics. J Thorac Oncol 2019;14:130-134.

29. Chalmers ZR, Connelly CF, Fabrizio D, et al. Analysis of 100,000 human cancer genomes reveals the landscape of tumor mutational burden. Genome Med 2017:9:34

30. Weickhardt AJ, Scheier B, Burke JM, et al. Continuation of EGFR/ALK inhibition after local therapy of oligoprogressive disease in EGFR mutant (Mt) and ALK+ non-small cell lung cancer (NSCLC) [abstract]. J Clin Oncol 2012;30(Suppl):Abstract 7526

31. Weickhardt AJ, Scheier B, Burke JM, et al. Local ablative therapy of oligoprogressive disease prolongs disease control by tyrosine kinase inhibitors in oncogene-addicted non-small-cell lung cancer. J Thorac Oncol 2012;7:1807-1814.

32. Gan GN, Weickhardt AJ, Scheier B, et al. Stereotactic radiation therapy can safely and durably control sites of extra-central nervous system oligoprogressive disease in anaplastic lymphoma kinase-positive lung cancer patients receiving crizotinib. Int J Radiat Oncol Biol Phys 2014;88:892-898

33. Borghaei $H$, Paz-Ares $L$, Horn $L$, et al. Nivolumab versus docetaxel in advanced nonsquamous non-small-cell lung cancer. N Engl J Med 2015;373:1627-1639.

34. Garassino MC, Cho BC, Kim JH, et al. Durvalumab as third-line or later treatment for advanced non-small-cell lung cancer (ATLANTIC): an openlabel, single-arm, phase 2 study. Lancet Oncol 2018;19:521-536. 